\title{
Régionalisation des pluies annuelles dans l'Algérie nord-occidentale
}

Regionalisation of annual rainfall in the north-western parts of Algeria.

Regionalisierung des Jahresniederschlags im Nordwesten Algerien

\section{Abderrahmane Medjerab et Latifa Henia}

\section{(2penEdition}

\section{Journals}

Édition électronique

URL : http://journals.openedition.org/rge/501

DOI : 10.4000/rge.501

ISSN : 2108-6478

Éditeur

Association des géographes de l'Est

Édition imprimée

Date de publication : 1 avril 2005

ISSN : 0035-3213

\section{Référence électronique}

Abderrahmane Medjerab et Latifa Henia, « Régionalisation des pluies annuelles dans l'Algérie nordoccidentale », Revue Géographique de l'Est [En ligne], vol. 45 / 2 | 2005, mis en ligne le 10 juin 2009, consulté le 07 septembre 2020. URL : http://journals.openedition.org/rge/501 ; DOI : https://doi.org/ $10.4000 /$ rge.501

Ce document a été généré automatiquement le 7 septembre 2020

Tous droits réservés 


\title{
Régionalisation des pluies annuelles dans l'Algérie nord-occidentale
}

\author{
Regionalisation of annual rainfall in the north-western parts of Algeria. \\ Regionalisierung des Jahresniederschlags im Nordwesten Algerien
}

Abderrahmane Medjerab et Latifa Henia

\section{NOTE DE L'ÉDITEUR}

Article reçu le 15 janvier 2005, accepté le 28 février 2005

1 La pluviométrie présente un intérêt majeur comme signature au-delà de son apport pour la définition du climat. Sa régionalisation s'avère très utile dans de nombreuses applications où il est nécessaire de minimiser la variabilité spatiale du paramètre analysé : détermination des "stations étalons », contrôle et reconstitution de données manquantes (Champeaux et al.,1996).

2 L'objectif d'une régionalisation (ou zonage) climatique est d'obtenir un découpage d'un territoire en zones homogènes, à l'intérieur desquelles le comportement climatique est similaire au(x) paramètre s) étudié(s). À cet effet, nous pouvons distinguer, dans la zone d'étude, plusieurs échelles spatiales emboîtées :

- une échelle microclimatique, de distance inférieure au kilomètre, qui représente en fait le parcellaire souvent confondue, par l'expression, avec l'échelle locale ;

- une échelle régionale, de distance caractéristique de l'ordre de 100 à 200 kilomètres, pour laquelle interviennent essentiellement la latitude, la distance à la mer, et le positionnement par rapport aux massifs montagneux, une échelle, appelée topoclimatique de l'ordre de 10 à 20 kilomètres ;

Les recherches entreprises sur le climat de l'Algérie par l'Agence Nationale des Ressources Hydrauliques et l'office National de la Météorologie, ont mis en évidence les premiers caractères du régime de la distribution géographique des précipitations par ACP seulement. Ce travail pose la même problématique, mais en utilisant deux 
techniques mathématiques complémentaires : l'Analyse en Composantes Principales et l'Analyse Factorielle Discriminante, en essayant toutefois d'interpréter le régime de la distribution géographique des pluies pour les régions homogènes mises en évidence.

\section{Choix et origine des données}

4 Notre choix s'est porté sur les données mensuelles des précipitations des 220 stations météorologiques dont l'altitude varie entre 20 et $1250 \mathrm{~m}$, pour une période de 48 ans (1942-1990). Nous considérons que la région d'étude dispose d'un réseau météorologique estimé important par rapport à l'Algérie orientale. L'inégale densité ressort clairement sur la figure 1 .

Figure 1 : Localisation des postes climatiques.

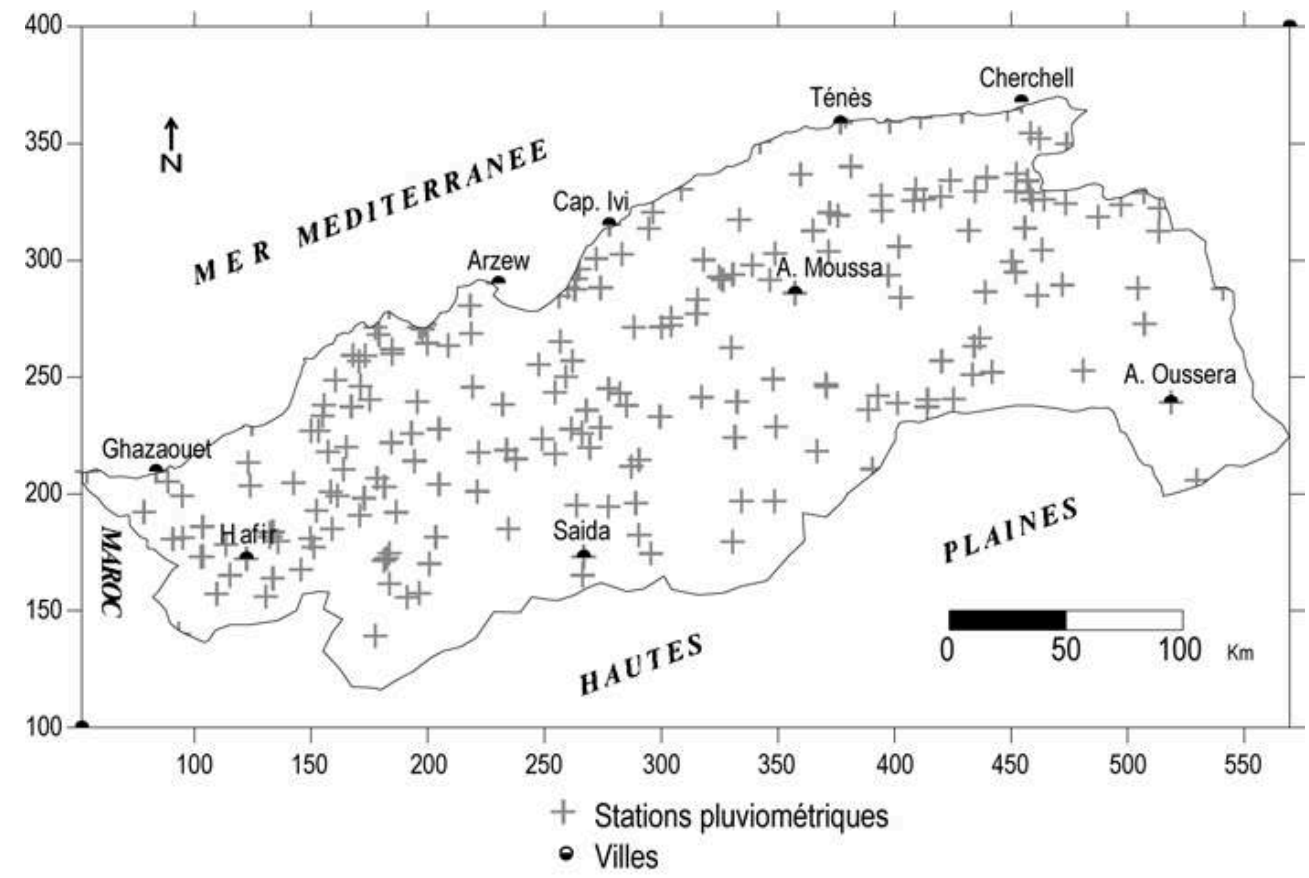

5 Sa densité est forte dans les plaines. Cela s'explique par la mise en valeur des terres agricoles des plaines qui a débuté avec l'arrivée des colons Français. Ce lien étroit entre les observations météorologiques et la mise en valeur des terres agricoles a donc été une contribution essentielle à la création d'un réseau météorologique, plus dense dans les plaines et faible dans les zones montagneuses. Les données choisies ont été obtenues par les archives de l'Agence Nationale des Ressources Hydrauliques (ANRH) et l'office National de la Météorologie (ONM), pour lesquelles nous avons effectué une critique systématique à l'échelle mensuelle (Medjerab, 2003). La période retenue dans l'étude, après analyse critique, correspond à une série concordante, sans lacune, suffisamment longue pour être traitée statistiquement. Ce choix et cette critique nous ont permis de constituer enfin une banque de données pluviométriques prête à tout traitement statistique. Elle se présente sous la forme d'un tableau avec deux entrées: l'une en lignes, ce sont les observations des 220 postes pluviométriques (individus); l'autre en colonnes, ce sont les variables représentées par les coordonnées géographiques des stations, l'altitude et les moyennes mensuelles. 


\section{Analyse multidimensionnelle des données}

6 Deux techniques mathématiques d'analyse multidimensionnelle ont été appliquées dans ce travail, à savoir : l'ACP et l'AFD.

\section{A. L'Analyse en composantes principales (ACP)}

7 L'Analyse en Composantes Principales sans rotation permet la description des données contenues dans un tableau individus / variables quantitatives ; c'est la méthode de base de l'analyse des données. Elle permet d'étudier les données en termes de corrélation, c'est- à-dire de détecter les stations ayant le même comportement (Maheras et al., 1991). Le grand avantage de cette technique réside dans son aptitude à traiter de façon simultanée un grand nombre de données. Elle permet, en outre, de dégager les interrelations complexes qui existent entre les variables et les résume ou les réduit en un petit nombre d'indicateurs appelés facteurs ou composantes principales. Elle est linéaire, car il s'agit d'une combinaison linéaire des variables de départ. L'application de l'algorithme de l'ACP sans rotation dans notre tableau a donné les résultats suivants (tableau 1) qui comprend des variances des trois premières composantes par rapport à la variance totale, ainsi que leur pourcentage cumulé.

Tableau 1 : Résultats de l'Analyse en Composantes Principales.

\begin{tabular}{|l|l|l|}
\hline Variance & \% de la variance & \% cumulé \\
\hline 5,5634 & 46,4 & 46,4 \\
\hline 2,3703 & 19,8 & 66,2 \\
\hline 0,8683 & 7,2 & 73,4 \\
\hline
\end{tabular}

D'après ce tableau, on constate que les trois premières composantes expliquent près de $80 \%$ de la variance totale, et nous n'avons donc que $20 \%$ de perte d'information.

- La première composante est bien corrélée avec 28 stations (contribution + 0,70) dont l'altiude varie entre 26 et $1270 \mathrm{~m}$. Cet axe manifeste un comportement d'ensemble, c'est-àdire qu'il traduit un effet de taille, car toutes les stations de fortes et de faibles coordonnées s'organisent sur le coté négatif de l'axe. Ce comportement d'ensemble dans le même sens explique la forte corrélation existant entre la plupart des stations.

- La seconde composante explique 19,8\% de la variance totale, elle est bien corrélée avec 10 stations se trouvant dans l'Est et l'Ouest de la région (contribution +60 ) dont l'altitude varie entre 82 et $1080 \mathrm{~m}$.

- La troisième composante principale explique 7,2 \% de la variance totale. Elle est bien corrélée avec 3 stations (contribution + 0,55) dont l'altitude varie entre 120 et $645 \mathrm{~m}$. Le choix de trois premières composantes a été fait d'une façon empirique.

Les stations à forte contribution, situées sur les versants nord de l'Atlas Tellien, se trouvent exposées «au vent " par rapport au flux dominant de l'W et NW. Pour une meilleure interprétation des résultats, on a tracé, sur la carte géographique de la région 
à l'échelle de $1 / 500000^{\mathrm{e}}$, les isolignes de contribution des variables avec les quatre composantes principales significatives (figure 2).

Figure 2a : Contribution des individus avec la première composante principale.

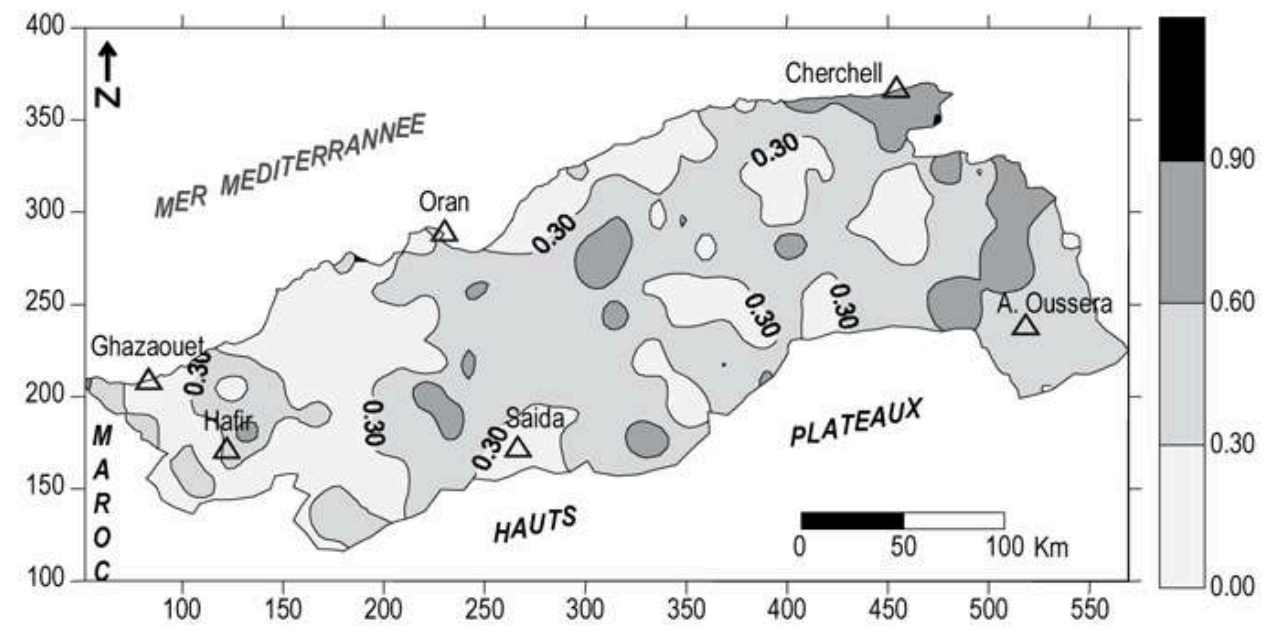

Figure $2 \mathrm{~b}$ : Contribution des individus avec la deuxième composante principale.

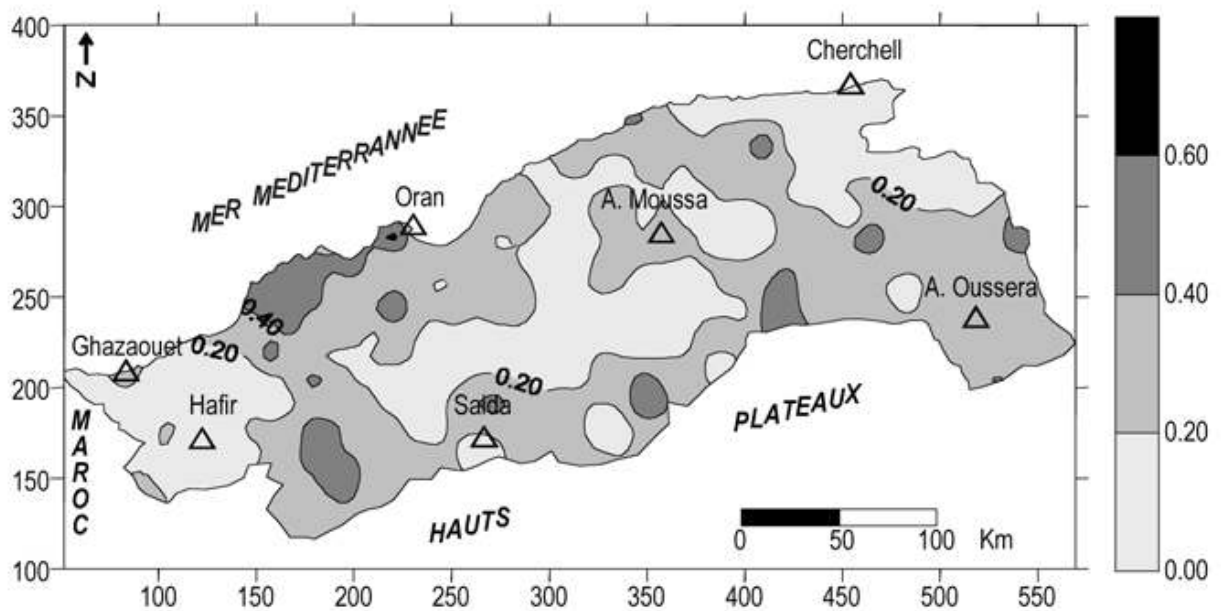


Figure $2 c$ :Contribution des individus avec la troisième composante principale.

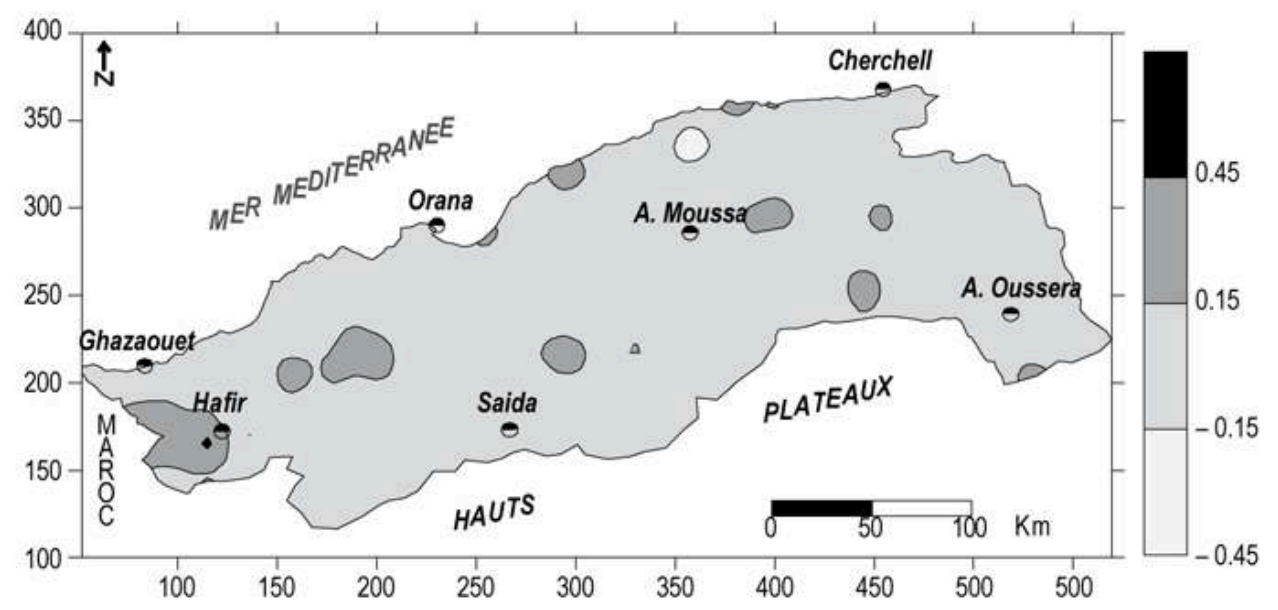

\section{B. Régionalisation des pluies par l'analyse des proximités}

10 Nous avons utilisé la dispersion des stations sur l'axe 1, pour pouvoir dégager des régions à pluviométrie distincte. Nous rappelons que l'axe 1 explique à lui seul près de $50 \%$ de la variance totale. Il sépare les stations en deux groupes, l'un du côté positif de l'axe, et l'autre du côté négatif. Le plan principal défini par les deux premiers axes, nous a permis d'effectuer une pré-régionalisation des postes en 4 régions pluviométriques (figure 3) dont les limites sont définies d'une façon empirique sur le plan (1-2). L'optimisation de cette classification empirique sera validée par l'AFD. Les régions définies par l'Analyse en Composantes Principales serviront de groupe de départ pour une optimisation maximum de la régionalisation. 
Figure 3 : Représentation des individus sur le plan factoriel (1-2).

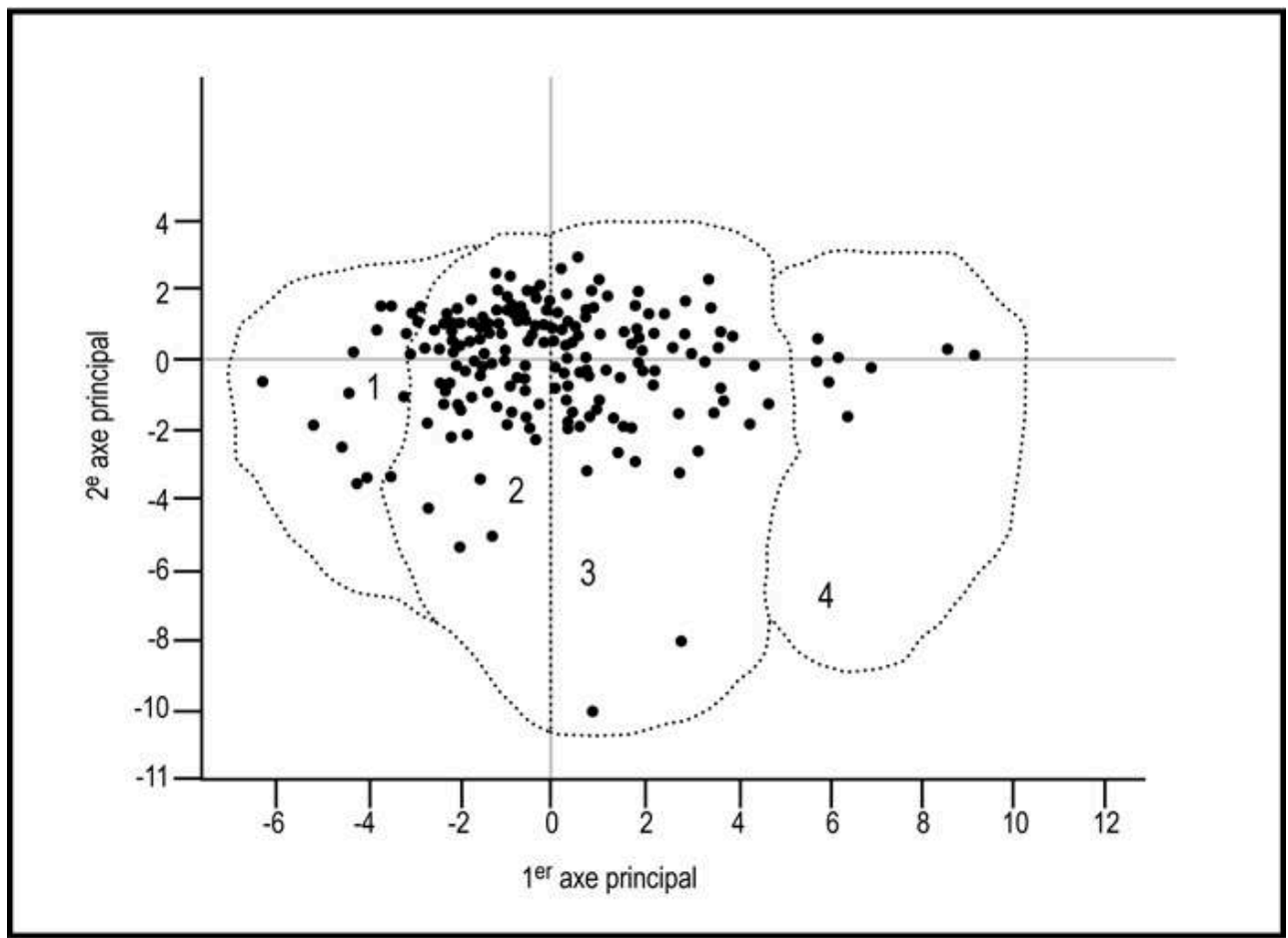

Conception et réalisation: A. Medjerab, L. Henia informatique (lllustrator) : S. Edelblutte.

\section{L'Analyse Factorielle Discriminante (AFD)}

11 L'Analyse Factorielle Discriminante est une méthode permettant de comparer des groupes d'individus à l'aide de plusieurs variables. Ces groupes peuvent contenir un nombre différent d'individus, dans un double objectif : d'abord de discrimination, c'està-dire de séparation des groupes; ensuite de classement, c'est-à-dire d'affectation d'individus à l'un des groupes. Cette affectation se fait par la méthode du plus proche voisin. Le but de l'utilisation de l'Analyse Factorielle Discriminante est de faire apparaître le mieux possible les frontières entre les principales régions pluviométriques (Zahir, 1994).

\section{Régionalisation des pluies annuelles par l'Analyse Factorielle Discriminante}

12 Afin de pouvoir valider les régions définies par l'ACP, l'Analyse Factorielle Discriminante est utilisée sur les cinq variables suivantes: les coordonnées géographiques, le total de la période humide (septembre à février) et la période sèche (mars-août), d'après une classification empirique, l'analyse de la variance par variable a donné les résultats figurant dans le tableau suivant (tableau 2).

Tableau 2 : Analyse par variable.

\begin{tabular}{|l|l|l|l|l|l|}
\hline Variables & $X$ & $Y$ & Altitude $(m)$ & Période humide & Période sèche \\
\hline
\end{tabular}




\begin{tabular}{|l|l|l|l|l|l|}
\hline Fc & 4,38 & 2,14 & 9,79 & 113,7 & 94,82 \\
\hline Probabilité (\%) & 0,53 & 9,47 & 0,00 & 0,00 & 0,00 \\
\hline
\end{tabular}

Il en ressort que la variable la plus significative est le total de la période humide (total hiver), et à un degré moindre, le total de la période sèche (total été). Pour justifier que la discrimination a été réalisée, nous avons calculé le pseudo-F des différentes variables discriminantes (tableau 3).

Tableau 3 : Variance et pseudo-F des variables discriminantes.

\begin{tabular}{|l|l|l|l|l|l|}
\hline Axe & Valeur propre & Inertie (\%) & Pseudo-F & Probabilité (\%) & Coef. Corrél. \\
\hline 1 & 2,5738 & 94,4 & 185,31 & 0,00 & 0,7202 \\
\hline 2 & 0,1460 & 5,8 & 10,51 & 0,08 & 0,1274 \\
\hline 3 & 0,0060 & 0,2 & 4,3 & 1,28 & 0,0059 \\
\hline
\end{tabular}

14 D'après ce tableau, on constate que le pseudo-F de la première variable discriminante est largement plus grand que le plus grand des F du tableau 2 (185,31 contre 10,51 et $4,3)$, donc la discrimination semble parfaitement justifiée. D'autre part, on peut constater que la première variable explique plus de $94 \%$ de la variance totale, ce qui implique que le premier axe regroupe presque la totalité de l'information. C'est pour cela que nous n'analyserons que les résultats du premier facteur discriminant. Les résultats ainsi définis sont représentés dans le tableau suivant (tableau 4).

Tableau 4 : Coordonnées et qualité de la représentation des variables sur l'axe 1.

\begin{tabular}{|l|l|l|l|l|l|}
\hline Variables & $X$ & $Y$ & $Z(m)$ & Période humide & Période sèche \\
\hline \hline Fc & 0,3396 & 0,9259 & 0,6246 & 0,9949 & 0,9905 \\
\hline \hline Probabilité (\%) & 0,1153 & 0,8572 & 0,3901 & 0,9898 & 0,9811 \\
\hline
\end{tabular}

15 Nous remarquons d'après ce tableau que la coordonnée $Y$, et la période humide (total hiver) et la période sèche (total été) sont bien représentées (total hiver ayant le plus fort coefficient de corrélation: 0,98). L'analyse des appartenances des stations a été effectuée par la distance de $\mathrm{D}^{2}$ de Mahalanobis. Les résultats sont données dans le tableau suivant (tableau 5).

Tableau 5 : Distance de D2 de Mahalanobis entre les différents groupes.

\begin{tabular}{|l|l|l|l|l|}
\hline Groupe & 1 & 2 & 3 & 4 \\
\hline \hline 1 & 0,0000 & & & \\
\hline
\end{tabular}




\begin{tabular}{|l|l|l|l|l|}
\hline 2 & 2,2395 & 0,0000 & & \\
\hline 3 & 2,9233 & 1,1922 & 0,0000 & \\
\hline 4 & 4,5522 & 1,1649 & 1,9989 & 0,0000 \\
\hline
\end{tabular}

De cette matrice des distances, on peut constater que les groupes les plus éloignés sont les groupes 1 et 4 , et les plus proches sont 2 et 3 . Le groupe 1 est le plus éloigné de tous les groupes. 41 stations ont changé de groupe entre l'Analyse en Composantes Principales et l'Analyse Factorielle Discriminante. Le plus important changement s'est fait entre le groupe 1 et 2 et le groupe 2 et 3 . A partir de ces résultats, on peut supposer que ces groupes ont de nombreux traits communs, les régions définies après AFD sont données par la figure 4 .

\section{Analyse et interprétation de la répartition spatiale des pluies par région}

\section{A. Région I}

La région I est localisée à l'Est (figure 4), dans les hautes plaines, et le plateau du Serssou, (région de Ain- Oussera, Ksar el Boughari) alors qu'à l'Ouest elle regroupe la région de Ras- El-Ma. Toutes ces régions sont relativement élevées (580-1 $194 \mathrm{~m}$ ), et abritées, par rapport aux courants pluvieux intéressant l'Algérie nord- occidentale. La région I se caractérise par l'indigence de la pluviosité.

Figure 4 : Régionalisation des pluies annuelles par l'AFD.

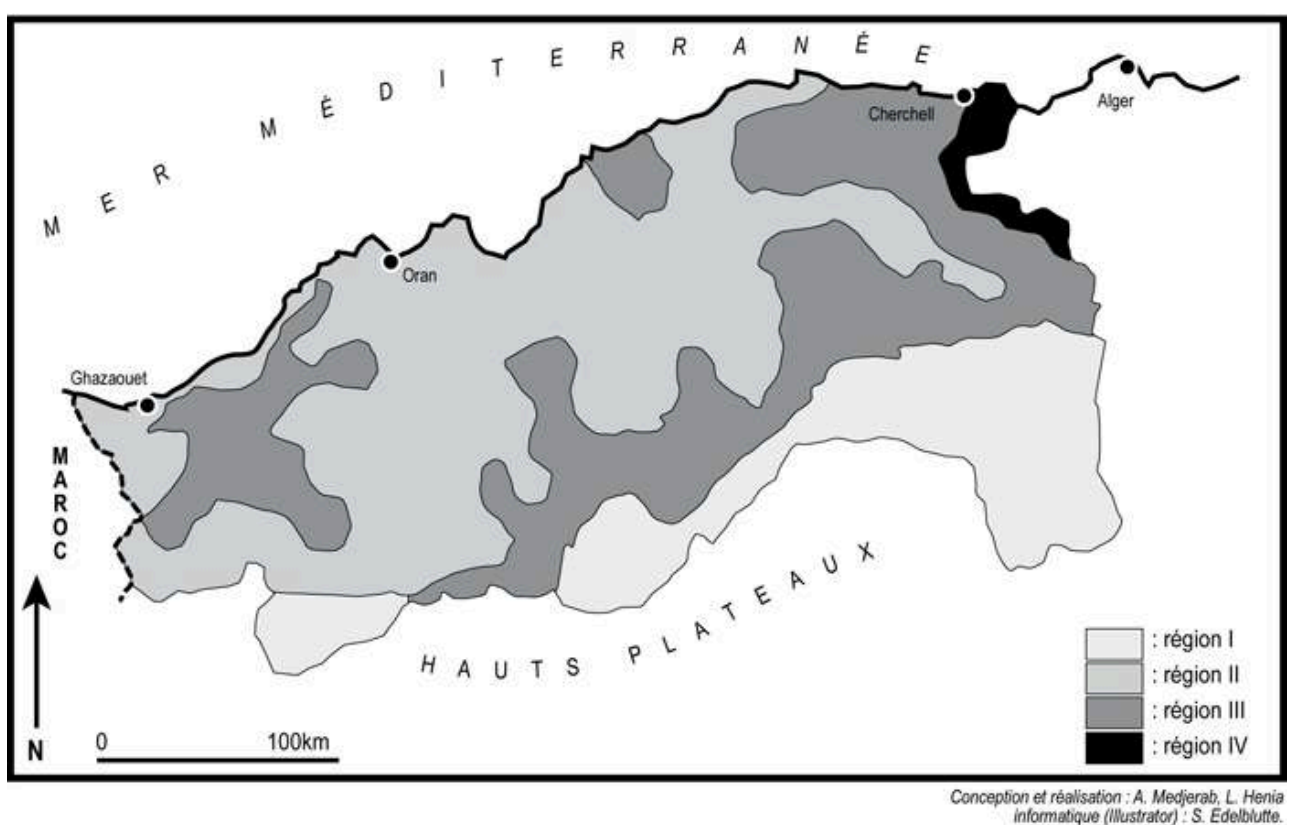

En effet, partout la moyenne est inférieure à $400 \mathrm{~mm}$, elle tombe à $199 \mathrm{~mm}$ à Zoubiria. De même, le nombre de jours de pluie est le plus faible de la zone d'étude, il est partout inférieur à 67 jours en moyenne par an, et tombe à moins de 43 jours à la station de 
Ain-Oussera. Le caractère des pluies demeure très irrégulier, le coefficient de variation le plus fort est observé dans la région de Rosfa avec 0,51. Le régime des pluies se distingue par un régime continental, le maximum se place en automne et au printemps. Il constitue 32 à $40 \%$ du total annuel de cette région, où l'été est partout sec. Cette nuance se caractérise par la fréquence de fortes intensités, qui tombent sous forme de pluies torrentielles ( $75 \mathrm{~mm}$ pluies maximales journalières enregistrées pendant l'année 1930-1931 dans la station de Khemisti). Le maximum automnal est lié aux orages qui abordent la région par le Sud et le Sud-Ouest pendant cette saison, alors que le minimum est enregistré en hiver et que les pluies, pendant cette saison, sont générées en grande partie par un courant d'W à perturbé le type de circulation de l'Ouest et Nord-Ouest. Or cette région est abritée par rapport aux influences de ces flux pluvieux.

\section{B. Région II}

19 Elle est localisée surtout à l'Ouest et au Centre. Elle se caractérise par une position d'abri relatif par rapport aux flux pluvieux de l'Ouest et du Nord-Ouest, et par le faible volume du relief. Dans cette région, les moyennes annuelles sont partout inférieures à $500 \mathrm{~mm}$; en effet, dès qu'on dépasse les monts de l'Atlas Tellien vers le Sud, et le SudOuest, la pluviométrie diminue : $480 \mathrm{~mm}$ à Oued Berkeche, $336 \mathrm{~mm}$ à El Hacaiba, mais à l'Est elle passe de $425 \mathrm{~mm}$ à Sidi M'hamed Ben Ouda, à $289 \mathrm{~mm}$ à Saline Oued Djama. Le coefficient de variation annuel est inférieur à 0,21 dans la plupart des stations. Cette variabilité est plus faible dans le Nord-Ouest $(0,21-0,41)$ à moyennement faible à l'Est $(0,23$ et 0,59$)$. Le régime pluviométrique se caractérise à l'échelle mensuelle par un double maximum pluviométrique : l'un se place en décembre ou janvier, l'autre se place en février dans l'Atlas Tellien, et au printemps dans les hautes plaines. Le maximum hivernal, est lié à la fréquence du type de circulation de l'Ouest et du Nord- Ouest $(50,4 \%$ et $36,9 \%)$. Dans cette région, l'influence de la continentalité apparaît nettement dans certaines zones. Cela s'explique par une double opposition :

- une position d'abri par rapport aux influences des flux pluvieux de direction Ouest et Nord-

Ouest ;

- une position d'abri relatif par rapport aux influences de la mer.

Cette nuance continentale apparaît d'avantage à l'Ouest, au Centre, et à un degré moindre à l'Est. L'influence de la continentalité dans ces régions se traduit surtout par une faible pluviométrie en saison chaude qui représente seulement $40 \%$ du total annuel au Sud (hautes plaines), 30 à $32 \%$ au Nord-Ouest et 27 à $31 \%$ au Nord-Est.

\section{Région III}

La région III englobe les zones montagneuses ainsi que les hautes plaines intérieures. Elle regroupe les Monts de Dahra au Nord-Est (Dj. El Gourine $736 \mathrm{~m}$, au Nord de Menaceur et Dj. Arbal $1095 \mathrm{~m}$ et Dj. Zeccar $1576 \mathrm{~m}$ à l'Est), les monts de Terraras (1 021 $\mathrm{m})$, les monts de Tessala (1 061m. ), les monts de Tlemcen (1 381m) à l'Ouest, les monts de Saida $(1043 \mathrm{~m})$ au Sud-Ouest et enfin le versant Sud du massif de l'Ouarsenis (Dj. Amrane. 1520 m., Dj. Zerloul 1075 m) au Sud-Est (figure 5). 


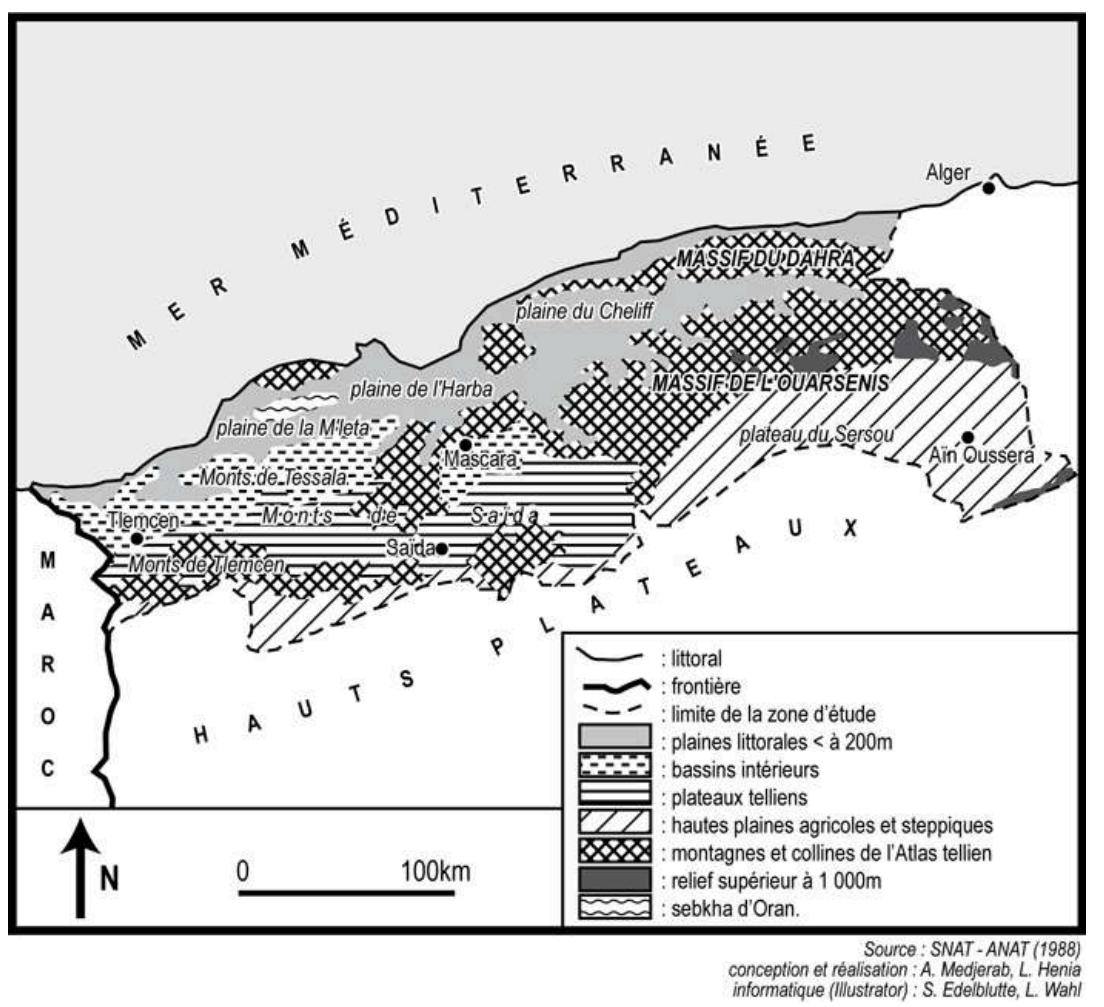

Toutes les zones regroupées dans la région III, se caractérisent par l'importance relative des totaux pluviométriques, dont la moyenne annuelle dépasse $500 \mathrm{~mm}$; elle est de $556 \mathrm{~mm}$ à Derrag, $573 \mathrm{~mm}$ à Damous, $582 \mathrm{~mm}$ à Gouraya, $581 \mathrm{~mm}$ à Cherchell, $614 \mathrm{~mm}$ à Tlemcen, $819 \mathrm{~mm}$ à Sidi-Medjahed. L'importance relative des précipitations dans cette région est en étroite relation avec sa position par rapport aux courants pluvieux. Ainsi, le type de temps le plus fréquent qui intéresse la région est OuestNord-Ouest (50,4\%, 36,9\%), Nord (4\%) et retour d'Est (9,2\%). Cependant, la position en latitude crée des nuances à l'intérieur de cette région avec l'organisation spatiale suivante :

-à l'Ouest, les monts de Tlemcen qui constituent le premier écran montagneux abordé par les courants de l'Ouest et Nord-Ouest, connaissent une moyenne annuelle comprise entre $575 \mathrm{~mm}$ à Ain-Essouk, et $627 \mathrm{~mm}$ à Tlemcen ;

- à l'Est, les monts de Zaccar, Dahra, Khemis Miliana constituent le premier obstacle orographique abordé par les courants du Nord-Ouest avec une moyenne annuelle comprise entre $573 \mathrm{~mm}$ (station de Damous) et $634 \mathrm{~mm}$ (station de Sidi-Ghiles);

- au Sud, les monts de Saida, Tiaret, enregistrent $412 \mathrm{~mm}$ à Saida, et $454 \mathrm{~mm}$ à Mellakou.

Cette région III se caractérise par une relative régularité de la pluviosité. En effet, le coefficient de variation annuel ne dépasse pas 0,27 pour la plupart des stations, tombant même à 0,19 à El-Khemis, alors qu'il peut atteindre 0,51 à Chlef dans la région II. À l'intérieur de la région III, le coefficient de variation est moins fort à l'Ouest (station de Lalla Setti 0,26; Ain Essouk 0,23) qu'à l'Est (Damous 0,34). Cette relative régularité des pluies est en rapport avec la bonne exposition face aux courants perturbés d'Ouest à Nord-Ouest, puisqu'il s'agit du type de circulation le plus fréquemment observé et le plus pluvieux. Le régime pluviométrique se caractérise par un maximum qui s'observe pendant la saison froide (hiver), et l'automne est plus 
pluvieux que le printemps, mais l'été est sec. D'autre part, on distingue aussi des nuances entre les hautes plaines et l'Atlas Tellien, avec des pluies lors de la saison chaude (printemps-été) qui représentent entre 31 et $39 \%$ du total annuel dans les hautes plaines. Le pourcentage arrive à $56 \%$ du total annuel dans la partie Ouest de l'Atlas Tellien et $47 \%$ dans l'Est (Teniet El-Had).

\section{Région IV}

Cette région, surtout représentée dans l'Est de l'Atlas Tellien, regroupe les régions de Beni-Chicao, Médéa, Sidi- Medjahed, et elle s'organise sous forme d'îlots dans la partie Ouest de l'Atlas tellien (région de Sebra et Mefrouche). Toutes ces régions se caractérisent par l'importance relative des totaux pluviométriques, la moyenne annuelle dépassant $488 \mathrm{~mm}$, pour l'ensemble de la région. Elle est de $579 \mathrm{~mm}$ à BeniChicao, $854 \mathrm{~mm}$ à Média, $638 \mathrm{~mm}$ à Bordj- Bounama. L'importance relative des pluies dans cette région est en étroite relation avec leur position par rapport aux courants pluvieux. En effet, le type de temps le plus fréquent dans cette région est le type de temps de Nord dans la région de Médéa, Bordj-Bounama et Nord-Ouest dans la région de Bensakrane-Mefrouche. Ces types s'accompagnent de pluies importantes, et sont considérés comme les plus pluvieux.

La bonne exposition au Nord et Nord-Ouest est un facteur qui engendre une pluviométrie irrégulière par rapport aux autres régions, avec un coefficient de variation annuelle ne dépassant pas 0,31 dans la plupart des stations. Le régime pluviométrique présente un maximum observé pendant la saison froide (automnehiver), et un minimum en été. Le printemps enregistre une diminution des précipitations. Par ailleurs, des nuances se distinguent dans cette région, car les précipitations de la saison chaude (printemps + été) ne représentent que 19 à $20 \%$ du total annuel, dans les régions de l'Ouest (Ben sakrane, Mefrouche). Dans les régions situées à l'Est (Médéa, Ben Chicao) il représente 30 à $35 \%$. Cette région se caractérise également par:

- des intensités pluviométriques fortes, les pourcentages les plus forts sont observés dans les stations de montagne, et particulièrement celles de l'Est de l'Atlas Tellien ou elle est matérialisée par la faiblesse relative du nombre de jours de pluie. Ainsi on comptabilise 91 jours avec précipitations pour un total annuel de $854 \mathrm{~mm}$ à Médéa et 61 jours à Hafir pour un total de $735 \mathrm{~mm}$;

- l'importance des totaux pluviométriques, une certaine régularité de la pluviosité et un régime méditerranéen à maximum hivernal, avec néanmoins des nuances entre l'Est et l'Ouest.

\section{Conclusion}

Un des avantages les plus significatif de la méthode de l'Analyse en Composantes Principales et de l'Analyse Factorielle Discriminante est la possibilité de faire le groupement des stations météorologiques d'une région sans aucune hypothèse a priori, dans le cas où le pourcentage de la variance totale exprimée par les composantes principales significatives est très élevé (dans ce cas il est de $80 \%$ ). Les méthodes de l'Analyse multidimensionnelle, à savoir l'Analyse en Composantes Principales et l'Analyse Factorielle Discriminante, sont un puissant outil pour la mise en évidence de 
régions pluviométriques homogènes. Ces méthodes deviennent plus efficaces lorsqu'elles sont utilisées de façon complémentaire. C'est ainsi que quatre régions sont déterminées :

- la première région pluviométrique est la moins arrosée du fait d'un effet d'abri et de l'influence modérée de l'altitude et de la continentalité. Le rythme des pluies est très irrégulier. Le régime est continental, les maxi mas se placent pendant l'automne et le printemps;

- la seconde région est plus arrosée que la première, les moyennes annuelles sont partout supérieures à $400 \mathrm{~mm}$, le coefficient de variation est inférieure à 0,21 , le régime pluviométrique se caractérise à l'échelle mensuelle par un double maximum : décembre ou janvier ;

- la troisième région se caractérise par l'importance relative des totaux pluviométriques, plus de $500 \mathrm{~mm}$, et par une relative régularité de la pluviosité. Le coefficient de variation est de 0,19 , le régime pluviométrique se caractérise par un maximum qui se situe pendant la saison froide ;

- la quatrième région est la plus arrosée. Elle exprime l'influence simultanée de l'orographie et de la circulation atmosphérique régionale durant toute l'année. La moyenne annuelle atteint $800 \mathrm{~mm}$, le régime pluviométrique se caractérise par un maximum pendant la saison froide, et par des intensités pluviométriques relativement fortes.

27 L'influence de l'altitude sur la distribution géographique des précipitations est variée. Il y a une hausse des précipitations en fonction de l'altitude dans les stations exposées aux flux pluvieux, avec une variation du gradient de l'Ouest vers l'Est. Quoi qu'il en soit, l'altitude n'est pas toujours le facteur le plus important dans la distribution géographique des précipitations. D'autres facteurs tels que la distance de la station par rapport au sommet, la position du relief par rapport à la mer, la morphologie de l'orographie jouent aussi un rôle important.

\section{BIBLIOGRAPHIE}

BELLICHI A. (1985). - «Les températures maximales d'été dans le Maroc nord- occidental, essai d'explication du caractère général de variation », Revue Géographique du Maroc, $\mathrm{n}^{\circ}$ 9, nouvelle série, p. 54-72.

BENABDERRAHMANE Z., BENFARES A., MOKRANE B. (1994). — « Régionalisation des pluies de la région constantinoise ». In : Compte-rendu des journées de Statistique appliquée, Alger, Sidi-Frédj, 26 p.

CHAMPEAUX J.-L., PERIS P. (1990). - « Zonage du Sud-Ouest de la France à partir des températures minimales et maximales », Publications de l'Association Internationale de Climatologie, vol. 3 , p. 207-215.

CHAMPEAUX J.-L., TAMBURINI A. (1996). - « Zonage climatique de la France à partir des séries de précipitations (1971-1990) du réseau climatologique de l'État », La Météorologie, série 8, n 14, p. 4-44. 
HENIA L. (1980). - « Climat et bilan de l'eau en Tunisie, essai de régionalisation climatique par les bilans hydriques », Publications de la Faculté des Sciences Humaines et Sociales de Tunis, série géographie, vol. XXVI, $391 \mathrm{p}$.

HENIA L. (1980). - «Les précipitations pluvieuses dans la Tunisie Tellienne »,Publications de la Faculté des Lettres et Sciences Humaines de Tunis, deuxième série Géographie, vol. XIV, 262 p.

MEDJERAB A. (1998). - Étude pluviométrique de l'Algérie nord-occidentale; approche statistique et cartographie automatique, Thèse de doctorat d'État, Faculté des Sciences de la Terre, de Géographie et Aménagement du Territoire, Université des Sciences et de la Technologie Houari Boumediene, Alger, en préparation.

MAHARAS P. et al. (1991). - " Analyse en composantes principales des précipitations en Albanie ", Publications de l'Association Internationale de Climatologie, vol. 4, p. 155-161.

SERRA C., PERACIO M.-C. et al. (1993). - « Un zonage pluviométrique de la Catalogne obtenue à partir des précipitations enregistrées en 24 heures au printemps », Publications de l'Association Internationale de Climatologie, vol. 6, p. 276-283.

ZAHIR I., KHELIL N. (1994). - Régionalisation des crues du Rif Ouest Marocain, Mémoire de fin d'études, Institut de Génie Civil, Université des Sciences et de la Technologie Houari Boumediene, Alger, $108 \mathrm{p}$.

\section{RÉSUMÉS}

L'application de l'Analyse en Composante Principale (ACP), sans rotation, sur les précipitations mensuelles de 220 stations (1942-1990) implantées dans l'Algérie nord-occidentale relève les régions pluviométriques homogènes correspondant aux trois composantes principales significatives. Les régions obtenues sont validées par l'Analyse Factorielle Discriminante (AFD). L'examen de l'influence des différents paramètres physiques sur la distribution des précipitations, pour chaque région homogène, a montré que la plupart des facteurs ont une part dans la régionalisation.

We use monthly data or rainfall taken from 220 stations (1942-1990) in the North Western Algeria. The application of the principal components analysis without rotation show on the monthly data of rainfall, taken from 220 stations (1942-1990) in the North Western Algeria, schwas the obtained regions are validated by the Factorial Discriminatory Analysis (FDA). The exam of the different physical parameter's on the distribution of rainfall for the homogenous rainy regions, its schwa within influence on the regionalization.

Die Anwendung einer Hauptkomponenteanalyse (ACP), ohne rotation, auf die monatliche Niederschlagssummen von 220 Stationen (1942-1990) im Nord-West von Algerien zeigt homogene Niederschlagsgebiete welche die drei markanten Hauptkomponenten folgen. Die Gebiete sind durch eine AFD geprüft worden. Die Analyse des Einfluss von verschiedenen physikalische Parametern auf die Niederschlagsverteilung, für jedes homogenes Gebiet, zeigt dass die meisten Faktoren eine Wirkung auf die Regionalisierung haben. 
INDEX

Schlüsselwörter : Hauptkomponenteanalyse (ACP), Niederschlag, Nord-West Algerien

Mots-clés : Algérie nord-occidentale, méthode statistique, pluie annuelle, régionalisation

Keywords : North Western Algeria, rainfall year, regionalization, statistical method

\section{AUTEURS}

\section{ABDERRAHMANE MEDJERAB}

Faculté des Sciences de la Terre, de Géographie et Aménagement du Territoire, Université des Sciences et de la Technologie Houari Boumediene, BP 32 El Alia Bab Ezzouar, 16111 Alger, Algérie

\section{LATIFA HENIA}

Université de Tunis, Faculté des Sciences Humaines et Sociales, 94 bd. du 9 avril 1938, 1007 Tunis, Tunisie 